\title{
Application of Support Vector Machine Based on Particle Swarm Optimization in Low Voltage Line Loss Prediction
}

\author{
Tan Min ${ }^{*}$, Wang Xinghua1 ${ }^{1}$, Li Qing ${ }^{1}$, Guo Lexin ${ }^{1}$, Yu Tao , Feng Yongkun² \\ ${ }^{1}$ South China University of Technology, Guangzhou, China \\ ${ }^{2}$ Hunan Electric Power Transmission Construction Company, Changsha, China \\ *tanminscut@126.com
}

Keywords: support vector machine; particle swarm optimization; line loss prediction

\begin{abstract}
As low voltage line loss calculation is the difficulty of line loss, accurate prediction of low voltage line loss rate can guide energy-saving and consumption-reducing work effectively. In this paper, the support vector machine (SVM) Parameters Optimization Algorithm based on particle swarm optimization (PSO) is used to predict the low voltage line loss rate. After the analysis of the related factors that affecting line loss, the power supply; average length of lines; average capacity of transformers and maximum load are selected as parameters for the training of SVM prediction model, then use the line loss prediction model to predict the low voltage line loss rate. Predict results of model testing which uses part of the known data in typical year show the average prediction error is only $1.92 \%$, which can give a strong support for this line loss prediction model.
\end{abstract}

\section{Introduction}

Power loss is an important economic and technical indicator for the measurement of management level of power grid enterprise. From the perspective of voltage level, power loss can be divided into three parts, such as high-voltage loss, medium-voltage loss and low-voltage loss [1]. At present, there are mature theoretical analysis methods for high-voltage and medium-voltage power loss. Since the complex low-voltage grid structure and the numerous feeders increase the difficulty of power loss forecasting, no mature and scientific analysis forecast methods is relatively capable for low-voltage power loss. However, low-voltage power loss account for a large proportion in the line loss of power supply enterprise. So, analysis and research on low-voltage power loss is more critical.

For a long time, power grid company attaches great importance to the work of energy-saving and consumption-reducing. Although loss reduction methods of the high-voltage power grid is mature, due to the differences between the structure of high-voltage power grid and low-voltage power grid, distribution network. So line Loss analysis method of main network is not applicable to the distributed network. In summary, this paper introduce the support vector machine (SVM) based on particle swarm optimization(PSO) for line loss forecasting, which can grasp the key factors affecting the low-voltage line loss rate and guide the work of energy-saving and consumption-reducing.

\section{Support Vector Machine Based on Particle Swarm Optimization A.Principle of PSO algorithm}

PSO algorithm views the potential solutions of the optimization problem to be solved as a bird with no mass and volume, which was defined as particle. Particles fly in a D-dimensional space. In the flight of each particle, particle update its location according to the optimal solution found by the particle itself currently and the optimal solution found by the entire population so far. By constantly updating of the location, particles can ultimately find the optimal solution [2-4].

The basic process of PSO algorithm shown in Figure 1:

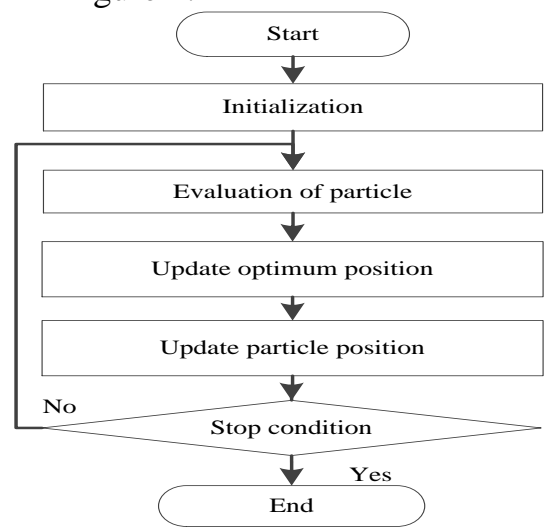

Fig.1 the flowchart of PSO algorithm 


\section{B Principle of SVM}

Support vector machine mapping the known parameters to high-dimensional space through kernel function, meanwhile constructs the optimal hyperplane in high-dimensional space. The representative sub-class of SVM is support vector regression (SVR). SVR has a linear output function, this feature of which can make it widely used in function fitting and forecast.

Non-linear SVR mapping input vectors to Hilbert space, followed by linear regression operation in the high-dimensional space, and finally get the nonlinear regression results of the original space [5-7].

\section{SVM parameters optimization based on PSO algorithm}

SVM parameters optimization based on PSO algorithm (PSO-SVM) uses PSO algorithm to optimize the parameters of SVM, which can reduce the time required for the calculation and improve the accuracy at the same time [8].

Before PSO-SVM the initial training samples are analyzed by principal component analysis method. Principal component analysis is a statistical method for dimension reduction [9]。Owning to the massive factors of line loss, besides correlations between all the different factors are different, so principal component analysis is used to filter the factors that have a greater impact on the line loss rate. After principal component analysis the loss forecast can reflect the actual situation more accurately.

The process of PSO-SVM algorithm shown in Figure 2:

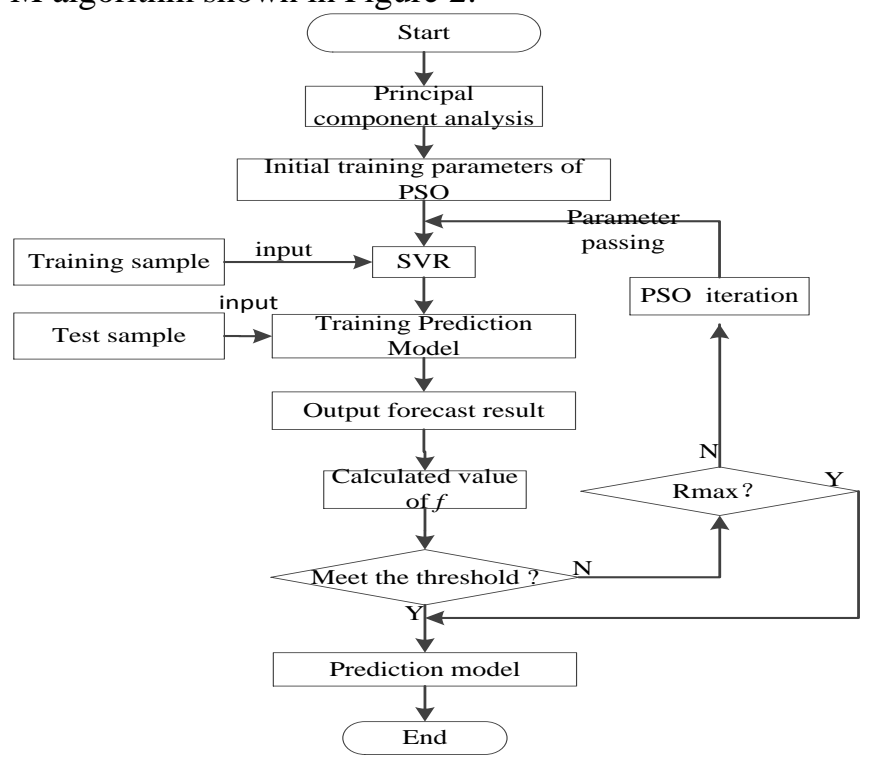

Fig.2 the flowchart of PSO-SVM algorithm

According to Fig.2, the process of PSO-SVM algorithm can be describe as follows: classify the sample collection into training sample set and test sample set; use the training sample set to train the prediction models; use test sample set to test the model. When the prediction error reaches the preset requirements or the maximum number of iterations, stops and outputs the final predictive model.

\section{PSO-SVM algorithm in low voltage line loss Prediction}

\section{A Prediction Model}

PSO-SVM use epsilon-SVR type and Kernel function is RBF. There are three parameters to be adjust, which are $\mathrm{c}, \mathrm{p}$ and $\mathrm{g}$. Where $\mathrm{c}$ is the penalty coefficient, representing the results of the tolerance of error; $\mathrm{p}$ is the loss coefficient of epsilon in epsilon-SVR type; $g$ is the parameters of basis function, which implicitly determines the distribution of the data mapped to the new feature space. PSO algorithm is used to search the optimal combination of the three parameters.

The maximum number of iterations is set to 300 , the size of the population is 100 in PSO algorithm. The solution space of PSO algorithm is three-dimensional, three-dimensional coordinates of each particle representing a group of feasible solution of the training parameters of $c, p$ and $g$. Fitness function is used to test the predictive accuracy of the model, which determined by contrast relative error of predicted value and the actual value. Fitness function $\mathrm{f}$ (i) formula as follows:

$$
f(i)=\frac{1}{m} \sum_{j=1}^{m} \frac{\left|y_{i j}-y_{i j}^{\prime}\right|}{y_{i j}} \times 100 \%
$$

where $\mathrm{m}$ is the population size, which is equal to 100 ; yij is the real value of ine loss; yij' is the prediction value of line loss. 
Set the threshold of $\mathrm{f}$ (i) as $\varepsilon$. In the population iterative process, if the prediction accuracy of formula (1) is less than $\varepsilon$ or the maximum number of iterations reaches the set value, PSO algorithm iteration is terminated and outputs the PSO-SVM prediction model.

The speed iterative of PSO population formula as follows:

$$
V_{i}^{k+1}=w_{i} \cdot V_{i}^{k}+C_{1} \cdot r_{1} \cdot\left(p_{\text {besti }}-X_{i}^{k}\right)+C_{2} \cdot r_{2} \cdot\left(g_{\text {best }}-X_{i}^{k}\right)
$$

where $\mathrm{i}$ is the number of particle; $\mathrm{k}$ is the number of the current iteration of PSO algorithm; $\mathrm{V}$ is the particle velocity; $\mathrm{X}$ is the position of a particle; pbesti is the best position of particle $\mathrm{i}$; gbesti is the best position of the population; $\mathrm{rl}$ and $\mathrm{r} 2$ are random numbers, $\mathrm{r} 1 \in[0,1], \mathrm{r} 2 \in\left[\begin{array}{ll}0,1\end{array}\right] ; \mathrm{c} 1$ and $\mathrm{c} 2$ are learning factors, $\mathrm{c} 1=\mathrm{c} 2=2 ; \omega$ is inertia weight, $\omega=0.9$.

The location iterative of PSO population formula as follows:

$$
X_{i}^{k+1}=X_{i}^{k+1}+w_{p} \cdot V_{i}^{k+1}
$$

where $i$ is the number of particle; $k$ is the number of the current iteration of PSO algorithm; $V$ is the particle velocity; $X$ is the position of a particle; $\omega_{p}$ is the elasticity coefficient.

\section{B.Model Checking}

There are a variety of factors affect the actual grid line losses, meanwhile the relationship among the factors is nonlinear. The factors contain active power supply; reactive power supply; distribution transformer capacity; line parameters; reactive power compensation capacity; the average length of the line; transformer average capacity; the maximum load; number of overloaded line; number of overloaded transformers; weather; seasonal factors and so on.

Therefore, line loss calculation can be abstracted as a multiple regression analysis problem. The various factors affecting the line loss can be treat as arguments of the regression problem, the line loss can be view as the dependent variables of the regression problem. In order to reduce computational time of line losses prediction model, and avoid over-learning phenomenon, principal component analysis method was adopted to reduce the dimension of the sample variables. Through principal component analysis removing factors that have a smaller impact on low-voltage line loss, finally power supply; average length of the line; transformer average capacity; the maximum load; number of overloaded line and number of overloaded transformers were choose as input parameters of prediction model.

To verify the accuracy of the model established, according to the basic network parameters and the completion of low-voltage line loss rate in a area of the base year, the prediction model was established. The low-voltage line loss rate were calculated from the voltage below $10 \mathrm{kV}$. There are 18 power supply bureaus in the area, low-voltage line loss rate in base year of 10 power supply bureaus were choose as the sample collection, so the other 8 power supply bureaus were the test collection.

Prediction model was established upon the sample collection. Low-voltage line loss of the base year is known to us, so the comparison of reactive value and the prediction value of the low-voltage line loss in test model can test

\begin{tabular}{|c|c|c|c|c|}
\hline \multirow{2}{*}{$\begin{array}{l}\text { Power } \\
\text { bureaus }\end{array}$} & \multirow{2}{*}{ supply } & \multicolumn{2}{|c|}{ Line loss of base year $(\%)$} & Unit（\%) \\
\hline & & Actual value & Predictive $v$ & Forecast error \\
\hline 1 & & 6.09 & 6.2 & 1.93 \\
\hline 4 & & 8.77 & 8.66 & 1.26 \\
\hline 6 & & 7.82 & 7.96 & 1.75 \\
\hline 12 & & 4.75 & 4.84 & 1.98 \\
\hline 13 & & 8.64 & 8.48 & 1.86 \\
\hline 14 & & 6.56 & 6.7 & 2.21 \\
\hline 15 & & 10.96 & 10.72 & 2.23 \\
\hline 18 & & 8.02 & 8.19 & 2.15 \\
\hline \multicolumn{2}{|c|}{ Average error } & - & - & 1.92 \\
\hline
\end{tabular}
the accuracy of the prediction model established. The comparison result shown in table 1:

the maximum prediction error is $2.23 \%$, the minimum prediction error is $1.26 \%$, the average prediction error is $1.92 \%$. So the established model can predict the low-voltage line loss rate effectively.

\section{Prediction result analysis}

Based on the line loss prediction model and combine the key parameters of base year to predict the low-voltage line loss of the next 3 years. Line loss rate forecasting results are shown in Table 2: 
Table 2 low-voltage line loss rate prediction

\begin{tabular}{|c|c|c|c|c|c|c|c|}
\hline $\begin{array}{l}\text { Power } \\
\text { bureaus }\end{array}$ & \multicolumn{3}{|c|}{ supply Line loss of predictive year $(\%)$} & $\begin{array}{l}\text { Power } \\
\text { bureaus }\end{array}$ & \multicolumn{3}{|c|}{ supply Line loss of predictive year $(\%)$} \\
\hline 1 & 3.18 & 3.12 & 3.04 & 10 & 8.65 & 7.85 & 7.08 \\
\hline 2 & 12.31 & 10.86 & 9.56 & 11 & 7.48 & 7.36 & 7.23 \\
\hline 3 & 4.52 & 4.28 & 4.01 & 12 & 4.58 & 4.47 & 4.34 \\
\hline 4 & 7.76 & 7.55 & 7.27 & 13 & 8.54 & 8.41 & 8.28 \\
\hline 6 & 7.26 & 7.12 & 6.98 & 15 & 10.01 & 9.56 & 8.87 \\
\hline 7 & 10.78 & 9.47 & 8.23 & 16 & 7.23 & 7.14 & 6.97 \\
\hline 8 & 8.92 & 8.78 & 8.64 & 17 & 9.84 & 8.85 & 7.73 \\
\hline 9 & 8.83 & 8.69 & 8.56 & 18 & 7.78 & 7.65 & 7.51 \\
\hline
\end{tabular}

Based on the above results, we can draw the conclusion that low-voltage line loss are gradually decreasing for the acceleration pace of construction of power grids. However, low-voltage loss still at a high level. According to the conventional case of a grid voltage level line loss distribution can be that low voltage line loss is busy most of the loss of electricity, so the focus is still on reducing losses on $10 \mathrm{kV}$ and below voltage. According to the line losses distribution of different grid voltage level it can be learned that low-voltage line loss is the most of the power loss, therefore, the focus of energy conservation and loss reduction work remains in the low level voltage.

By predicting the line loss rate can predict the future trend of line loss in the next few years, which is beneficial to identify the direction and focus on loss reduction; develop effective loss reduction measures and accomplish the goal of line loss index effectively. From the prediction value of line loss of low-voltage it can be seen that the management improvement of the distribution network has a relatively large room. To better respond to the national energy loss reduction policy and call for carbon emission reduction, works like increasing marketing management, carrying out marketing inspection work; fight against illegal use of electricity and stealing work should be enforced strictly.

\section{Conclusion}

In this paper, support vector machine parameters optimization algorithm based on particle swarm optimization used in low-voltage grid line loss rate forecast has achieved a good prediction effect. Through the low voltage line loss rate forecast, which combine factors correction can grasp the line loss rate trend more accurately. Line loss prediction can instruct the development of measures for reducing energy loss, formulation of scientific loss reduction innovative goal and assurance of the completion of line loss reduction strategy index.

\section{References}

[1] Chen Jiangbin, Chen Jingsong. Estimate analysis of low voltage line loss of Harbin power bureau [J]. Heilongjiang electric power, 2005, 27(1):4-7.

[2] Guanzhao Cui, Limin Qin, Sha Liu. Modified PSO algorithm for solving planar graph coloring problem[J]. Progress in Natural Science, 2008(18):353-357.

[3] Xu Gang, Qu Jingping, Yang Zhitao. An Improved Adaptive Particle Swarm Optimization Algorithm[J]. Journal of South ChinaUniversity of Technology(Natural Science Edition), 2008, 36(9):6-10.

[4] Gao Haibing, Zhou Chi, Gao Liang. General Particle Swarm Optimization Model[J]. Chinese Journal of Computers , 2005, 28(12):1980-1987.

[5] Haoran Zhang, Zhengzhi Han , Rui Feng. Support Vector Machine-Based Nonlinear System Modeling and Control[J]. Journal of Systems Engineering and Electronics, 2003, 14(3):53-58.

[6] Tian Shengfeng, Huang Houkuan. A Simplification Algorithm to Support Vector Machines for Regression[J]. Journal of Software, 2002, 13(6):1169-1172.

[7] Qing Wu, Sanyang Liu , Leyou Zhang. Adjustable entropy function method for support vector machine[J]. Journal of Systems Engineering and Electronics, 2008, 19(5):1029-1034.

[8] Ren Jiangtao, Zhao Shaodong, Xu Shengcan. Simultaneous Feature Selection and SVM Parameters Optimization Algorithm Based on Binary PSO[J]. Computer Science, 2007, 36(6):179-182.

[9] Lin Heping, Yang Chen. Research and Analysis of Fuzzy Principal Component Analysis[J]. Aeronautical Computing Technique, 2006, 36(6):16-20. 